CERN-PH-TH/2004-103

$\mathrm{DCPT} / 04 / 56$

DESY-04-089

$\mathrm{IPPP} / 04 / 28$

KA-TP-04-2004

MADPH-04-1384

hep-ph/0406323

\title{
Extracting Higgs boson couplings from LHC data
}

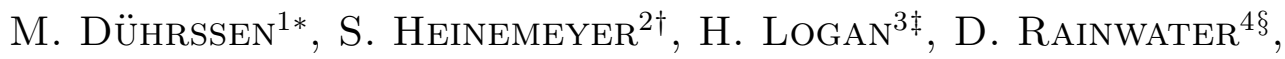 \\ G. WeIGLEIN ${ }^{5}$ AND D. ZEPPENFELD ${ }^{3,6 \|}$ \\ ${ }^{1}$ Physikalisches Institut, Universität Freiburg, Hermann-Herder-Str. 3, \\ D-79104 Freiburg, Germany \\ ${ }^{2}$ CERN TH Division, Dept. of Physics, CH-1211 Geneva 23, Switzerland \\ ${ }^{3}$ Dept. of Physics, University of Wisconsin, 1150 University Avenue, \\ Madison, WI 53706 USA \\ ${ }^{4}$ DESY Theory, Notkestr. 85, D-22603 Hamburg, Germany \\ ${ }^{5}$ Institute for Particle Physics Phenomenology, University of Durham, \\ Durham DH1 3LE, UK \\ ${ }^{6}$ Institut für Theoretische Physik, Universität Karlsruhe, D-76128 Karlsruhe, Germany
}

\begin{abstract}
We show how LHC Higgs boson production and decay data can be used to extract gauge and fermion couplings of Higgs bosons. We show that very mild theoretical assumptions, which are valid in general multi-Higgs doublet models, are sufficient to allow the extraction of absolute values for the couplings rather than just ratios of the couplings. For Higgs masses below $200 \mathrm{GeV}$ we find accuracies of $10-40 \%$ for the Higgs boson couplings and total width after several years of LHC running. Slightly stronger assumptions on the Higgs gauge couplings even lead to a determination of couplings to fermions at the level of $10-20 \%$. We also study the sensitivity to deviations from SM predictions in several supersymmetric benchmark scenarios as a subset of the analysis.
\end{abstract}

*email: michael.duehrssen@gmx.de

†email: Sven.Heinemeyer@cern.ch

†email: logan@pheno.physics.wisc.edu

§email: rain@mail.desy.de

ๆ email: Georg.Weiglein@durham.ac.uk

" email: dieter@particle.uni-karlsruhe.de 


\section{Introduction}

LHC experiments will have the capability to observe a Higgs boson, Standard Model (SM) or non, in a variety of channels, in particular if its mass lies in the intermediate mass region, $114<m_{H} \lesssim 250 \mathrm{GeV}$, as suggested by direct searches [1] and electroweak precision data [2]. Once the Higgs boson is discovered and its mass measured, one will want to gain as much information as possible on its couplings to both gauge bosons and fermions. These measurements will provide crucial tests of the mass generation mechanism realized in nature.

The various Higgs couplings determine Higgs production cross sections and decay branching fractions. By measuring the rates of multiple channels, various combinations of couplings can be determined. A principal problem at the LHC is that there is no technique analogous to the measurement of the missing mass spectrum at a linear collider [3] which would directly determine the total Higgs production cross section. The decay $H \rightarrow b \bar{b}$, which by far dominates for a light Higgs boson, will be detectable but suffer from large experimental uncertainties. In addition, some Higgs decay modes cannot be observed at the LHC. For example, $H \rightarrow g g$ or decays into light quarks will remain hidden below overwhelming QCD dijet backgrounds. This implies that absolute measurements of (partial) decay widths are possible only with additional theoretical assumptions.

One possible strategy was outlined in Refs. [4-6]. Assuming the absence of unexpected decay channels and a SM ratio of the $H \rightarrow b \bar{b}$ and $H \rightarrow \tau \tau$ partial widths, absolute measurements of $\Gamma(H \rightarrow W W, Z Z), \Gamma(H \rightarrow \tau \tau), \Gamma(H \rightarrow \gamma \gamma), \Gamma(H \rightarrow g g)$ and of the top quark Yukawa coupling squared, $Y_{t}^{2}$, are possible, with errors in the $10-30 \%$ range.

Here we revisit the information that could be extracted at the LHC from rate measurements of an intermediate-mass Higgs boson. We consider the expected accuracies at various stages of the LHC program: after $30 \mathrm{fb}^{-1}$ of low luminosity $\left(10^{33} \mathrm{~cm}^{-2} \mathrm{sec}^{-1}\right)$ running, $300 \mathrm{fb}^{-1}$ at high luminosity $\left(10^{34} \mathrm{~cm}^{-2} \mathrm{sec}^{-1}\right)$, and a mixed scenario where the weak boson fusion channels are assumed to suffer substantially from pile-up problems under high luminosity running conditions (making forward jet tagging and central jet veto fairly inefficient).

A rather model-independent analysis, where only ratios of couplings (or partial widths) could be extracted, was performed in Ref. [7]. Here we consider general multi-Higgs-doublet models (with or without additional Higgs singlets), in which the $H W W$ and $H Z Z$ couplings are bounded from above by their SM values, i.e., we impose theoretically motivated constraints on these two couplings. These constraints are valid, in particular, for the Minimal Supersymmetric Standard Model (MSSM), and will sharpen the implications of LHC data for Higgs couplings very significantly.

As an illustration of coupling measurements at the LHC, we consider specific MSSM benchmark scenarios of Ref. [8] as examples. The significance of deviations of the measured rates from SM predictions provides a measure of the sensitivity of LHC measurements in the Higgs sector.

The paper is organized as follows. In Section 2 we review the existing analyses on the production and decay channels used throughout our paper, followed by our theoretical assumptions and fitting procedure details in Sections 3 and 4 , respectively for general multiHiggs-doublet models and a specific MSSM scenario. Section 5 contains a summary and outlook. 


\section{Summary of Higgs boson channels}

In order to determine the properties of a physical state such as a Higgs boson, one needs at least as many separate measurements as properties to be measured, although two or more measurements can be made from the same channel if different information is used, e.g., total rate and an angular distribution. Fortunately, the LHC will provide us with many different Higgs observation channels. In the SM there are four relevant production modes: gluon fusion (GF; loop-mediated, dominated by the top quark), which dominates inclusive production; weak boson fusion (WBF), which has an additional pair of hard and far-forward/backward jets in the final state; top-quark associated production $(t \bar{t} H)$; and weak boson associated production $(W H, Z H)$, where the weak boson is identified by its leptonic decay. ${ }^{1}$

Although a Higgs is expected to couple to all SM particles, not all these decays would be observable. Very rare decays (e.g., to electrons) would have no observable rate, and other modes are unidentifiable QCD final states in a hadron collider environment (gluons or quarks lighter than bottom). In general, however, the LHC will be able to observe Higgs decays to photons, weak bosons, tau leptons and $b$ quarks, in the range of Higgs masses where the branching ratio (BR) in question is not too small.

For a Higgs in the intermediate mass range, the total width, $\Gamma$, is expected to be small enough to use the narrow-width approximation in extracting couplings. The rate of any channel (with the $H$ decaying to final state particles $x x$ ) is, to good approximation, given by

$$
\sigma(H) \times \mathrm{BR}(H \rightarrow x x)=\frac{\sigma(H)^{\mathrm{SM}}}{\Gamma_{p}^{\mathrm{SM}}} \cdot \frac{\Gamma_{p} \Gamma_{x}}{\Gamma},
$$

where $\Gamma_{p}$ is the Higgs partial width involving the production couplings and where the Higgs branching ratio for the decay is written as $\operatorname{BR}(H \rightarrow x x)=\Gamma_{x} / \Gamma$. Even with cuts, the observed rate directly determines the product $\Gamma_{p} \Gamma_{x} / \Gamma$ (normalized to the calculable SM value of this product). The LHC will have access to (or provide upper limits on) combinations of $\Gamma_{g}, \Gamma_{W}, \Gamma_{Z}, \Gamma_{\gamma}, \Gamma_{\tau}, \Gamma_{b}$ and the square of the top Yukawa coupling, $Y_{t}{ }^{2}$

Since experimental analyses are driven by the final state observed, we classify Higgs channels by decay rather than production mode, and then discuss the different production characteristics as variants of the final state. However, some initial comments on production modes are in order. First, experimental studies mostly do not yet include the very large $(\mathrm{N}) \mathrm{NLO}$ enhancements known for $g g \rightarrow H$ [9-11]. Even if background corrections are as large as for the signal, which they typically are not, the statistical significance of the GF channels will be greater than estimated by the current studies (which we have used for this paper). Furthermore, the NNLO calculations may reduce also the theory systematic uncertainty for the signal. Second, experimental studies do not consider WBF channels above $30 \mathrm{fb}^{-1}$ integrated luminosity, because the efficiency to tag forward jets at high-luminosity LHC running is not yet fully understood. This is a very conservative assumption. We also discuss a scenario where a higher luminosity is available in the WBF channels.

The literature on Higgs channels at LHC is extensive. We refer here only those analyses which we use in our fits. Mostly, these are recent experimental analyses which contain

\footnotetext{
${ }^{1}$ We do not consider diffractive Higgs production since its rate is in general small and also quite uncertain, which limits the usefulness of this channel for Higgs coupling determinations.

${ }^{2}$ We do not write this as a partial width, $\Gamma_{t}$, because, for a light Higgs, the decay $H \rightarrow t \bar{t}$ is kinematically forbidden.
} 
references to the earlier phenomenological proposals. We always use the rates as summarized in Ref. [7] for our fits. The individual channels used for the fits are:

- $\mathrm{GF} g g \rightarrow H \rightarrow Z Z[7,14]$

- $\mathrm{WBF} q q H \rightarrow q q Z Z[7]$

- $\mathrm{GF} g g \rightarrow H \rightarrow W W[7,14]$

- $\mathrm{WBF} q q H \rightarrow q q W W[22]$

- $W H \rightarrow W W W(2 l$ and $3 l$ final state) $[25,28]$

- $t \bar{t} H(H \rightarrow W W, t \rightarrow W b)(2 l$ and $3 l$ final state) [24]

- Inclusive Higgs boson production: $H \rightarrow \gamma \gamma[14]$

- WBF $q q H \rightarrow q q \gamma \gamma[17]$

- $t \bar{t} H(H \rightarrow \gamma \gamma)[29]$

- $W H(H \rightarrow \gamma \gamma)[29]$

- $Z H(H \rightarrow \gamma \gamma)[29]$

- $\mathrm{WBF} q q H \rightarrow q q \tau \tau[22,30,31]$

- $t \bar{t} H(H \rightarrow b \bar{b})[27]$

For the WBF channels we include a minijet veto, even if the study cited did not. In the discussion below, statements about Higgs rates typically refer to the SM-like case. Substantially suppressed branching ratios are possible beyond the SM and may change a measurement into an upper bound.

\section{$2.1 \quad H \rightarrow Z^{(*)} Z^{(*)} \rightarrow 4 \ell$}

Leptons are the objects most easily identified in the final state, so this decay is regarded as "golden" due to its extreme cleanliness and very low background. It is a rare decay due to the subdominance of $H \rightarrow Z Z$ relative to $H \rightarrow W^{+} W^{-}$, and because of the very small BR of $Z \rightarrow \ell^{+} \ell^{-}$. Fortunately, due to the possible decay to off-shell $Z$ bosons, a SM Higgs has non-negligible BR to $4 \ell$ even for $m_{H}<2 M_{Z}$, down to approximately $120 \mathrm{GeV} .{ }^{3}$ Due to the low event rate, current studies concentrate on inclusive measurements which are dominated by GF. They provide information mainly on the product $\Gamma_{g} \Gamma_{Z} / \Gamma$.

The most advanced analysis for this channel [12] was made recently by ATLAS. (For an older CMS study, see [13]). Its principal improvement over previous studies is the use of all available NLO results (the only study so far to do this) for both the dominant GF signal and its major backgrounds. Further improvements can be expected in the inclusion of off-shell contributions to the $g g \rightarrow Z Z$ background, for which ATLAS used an approximate K-factor.

By isolating the WBF contribution one obtains some independent information on the product $\Gamma_{W, Z} \Gamma_{Z} / \Gamma$, in particular if high-luminosity running can be exploited for this channel.

\footnotetext{
${ }^{3}$ We note that for such low masses, doubly off-shell effects must be taken into account.
} 


\section{$2.2 \quad H \rightarrow \gamma \gamma$}

Photons are also readily identifiable, but are more difficult than leptons to measure because of a large, non-trivial background from jets faking photons. Higgs photonic decay is loopinduced and therefore rare, even more so because of destructive interference between the top-quark and $W$ loops. This is in some sense advantageous, because this decay mode is thus sensitive to variations in the weak gauge and top Yukawa couplings and additional particles in the loop. This decay is visible in the SM only for the lower Higgs mass range, $110<m_{H}<150 \mathrm{GeV}$.

Despite the difficulties of identifying photons, which are not yet fully understood for the LHC, especially for high-luminosity running, Higgs decays to photons should be observable in both GF [14-16] and WBF [17,18], unless $\mathrm{BR}(H \rightarrow \gamma \gamma)$ is substantially smaller than in the SM. These channels measure the products $\Gamma_{g} \Gamma_{\gamma} / \Gamma$ and $\Gamma_{W, Z} \Gamma_{\gamma} / \Gamma$. The $H \rightarrow \gamma \gamma$ signals in $t \bar{t} H, W H$ and $Z H$ production $[14,19]$ are very weak, due to the paucity of events even at high-luminosity running, but could be used as supplemental channels, and would be especially useful if LHC observes a non-SM Higgs.

\section{$2.3 \quad H \rightarrow W^{+(*)} W^{-(*)} \rightarrow \ell^{+} \ell^{-}+\not p_{T}$}

This decay can be observed in GF $[14,20,21]$ and WBF $[22,23]$ using $W^{+} W^{-} \rightarrow \ell^{+} \ell^{-}+\not p_{T}$ final states, as well as in $t \bar{t} H$ associated production using combinations of multilepton final states [24]. The first two modes extract the products $\Gamma_{g} \Gamma_{W} / \Gamma$ and $\Gamma_{W}^{2} / \Gamma$ and are extremely powerful statistically, while the $t \bar{t} H$ mode can extract the top Yukawa coupling with high luminosity once $\Gamma_{W}$ is known. All these channels are accessible over a wide range of Higgs masses, approximately $120<m_{H}<200 \mathrm{GeV}$. An additional study [25] of the $W H, H \rightarrow$ $W W$ channel for $m_{H}>150 \mathrm{GeV}$ found only a very weak signal, less than $5 \sigma$ even for $300 \mathrm{fb}^{-1}$ of data. We include this channel in our analysis.

The GF mode should improve after (N)NLO effects are included, although the backgrounds considered did not include off-shell $g g \rightarrow W^{(*)} W^{*}$. Also, the single-top background was conservatively overestimated. A reanalysis of this channel with updated simulation tools would be in order.

\section{$2.4 \quad H \rightarrow \tau^{+} \tau^{-}$}

Observing Higgs decays to taus is not possible in GF because of serious background problems and because the invariant mass of a tau pair can be reconstructed only when the taus do not decay back-to-back, which leaves only a small fraction of GF events with sizable Higgs transverse momentum. Observation of $H \rightarrow \tau^{+} \tau^{-}$is possible in WBF, however [22, 26], for Higgs masses below about $150 \mathrm{GeV}$. As the average Higgs $p_{T}$ in this production mode is $\mathcal{O}(100) \mathrm{GeV}$, the taus are only rarely produced back-to-back. This is a relatively rare decay mode, since $\mathrm{BR}(H \rightarrow \tau \tau)$ is typically $5-10 \%$ in this mass region and the taus decay further. At least one tau must decay leptonically, giving another small BR. Fortunately, the QCD background to taus is small, due to excellent fake jet rejection. While not a discovery channel, this channel is statistically quite powerful even with only moderate luminosity, and thus becomes one of the more important decay modes in a couplings analysis. This channel measures the product $\Gamma_{W, Z} \Gamma_{\tau} / \Gamma$. 


\section{$2.5 \quad H \rightarrow b \bar{b}$}

Associated Higgs- $b$ quark production has too small a cross section in a SM-like Higgs sector to be observable, so the decay $H \rightarrow b \bar{b}$ is the only experimental access to the $b$ Yukawa coupling. Because this mode dominates Higgs decays at low mass $\left(m_{H} \lesssim 135 \mathrm{GeV}\right.$ within the SM), an accurate measurement of the bottom Yukawa coupling is extremely important. Unfortunately, due to the typically large QCD backgrounds for $b$ jets, it is very difficult to observe this decay. The production modes $t \bar{t} H[27,32,33]$ and $W H[14,34]$ might allow very rough measurements for such a light Higgs, but the statistical significances are quite low and the background uncertainties quite large and their rates probably underestimated; they are definitely high-luminosity measurements.

The $t \bar{t} H$ channel measures the product $Y_{t}^{2} \Gamma_{b} / \Gamma$, and so would require a separate, precise measurement of $Y_{t}$ to isolate $\Gamma_{b}$. For $W H$ production, the rate is proportional to $\Gamma_{W} \Gamma_{b} / \Gamma$. But here the $W b \bar{b}$ continuum background has hitherto been underestimated since the NLO QCD corrections are very large and positive [35]. A veto on additional jets may help but requires another detector-level simulation; unfortunately, it would also increase the background uncertainty because additional jet activity has been calculated at LO only. We include the $t \bar{t} H$ channel but not $W H$ in our analysis.

\subsection{Other channels}

The production and decay channels discussed above refer to a single Higgs resonance, with decay signatures which also exist in the SM. The Higgs sector may be much richer, of course. The MSSM with its two Higgs doublets predicts the existence of three neutral and one charged pair of Higgs bosons, and the LHC may be able to directly observe several of these resonances. Within SUSY models, additional decays, e.g., into very light super-partners, may be kinematically allowed. The additional observation of super-partners or of heavier Higgs bosons will strongly focus the theoretical framework and restrict the parameter space of a Higgs couplings analysis [36].

At the present time, even enumerating the possibilities is an open-ended task. For our present analysis we therefore ignore the information which would be supplied by the observation of additional new particles. Instead we ask the better-defined question of how well LHC measurements of the above decay modes of a single Higgs resonance can determine the various Higgs boson couplings or partial widths.

\section{Model assumptions and fits}

In spite of the many decay channels discussed above, the LHC is faced with the challenge that not all Higgs decay modes can be detected (e.g., $H \rightarrow g g$ is deemed unobservable) or that some important decay rates, in particular $H \rightarrow b \bar{b}$, will suffer from large experimental uncertainties. In a model-independent analysis, the limited information which will be available then will lead to strong correlations in the measurement of different Higgs couplings. These correlations mask the true precision of LHC measurements when the expected errors of particular observables like individual partial widths or branching ratios are considered.

The parameter correlations can be overcome by imposing theoretical constraints. One possible approach was suggested in Refs. $[4,5]$ : fixing the ratio $\Gamma_{b} / \Gamma_{\tau}$ to its SM value, the $H \rightarrow \tau \tau$ measurements can be used to pin down the poorly measured Higgs coupling to 
bottom quarks. Here we follow a different approach. We perform general fits to the Higgs couplings with the mildest possible theoretical assumptions, starting with the constraint

$$
\Gamma_{V} \leq \Gamma_{V}^{\mathrm{SM}}
$$

$(V=W, Z)$ which is justified in any model with an arbitrary number of Higgs doublets (with or without additional Higgs singlets). I.e., it is true for the MSSM in particular.

Even without this constraint, the mere observation of Higgs production puts a lower bound on the production couplings and, thereby, on the total Higgs width. The constraint $\Gamma_{V} \leq \Gamma_{V}^{\mathrm{SM}}$, combined with a measurement of $\Gamma_{V}^{2} / \Gamma$ from observation of $H \rightarrow V V$ in WBF, then puts an upper bound on the Higgs total width, $\Gamma$. It is this interplay which provides powerful constraints on the remaining Higgs couplings, allowing for their absolute determination, rather than simply ratios of their magnitudes.

\subsection{Fitting procedure}

Our analysis of expected LHC accuracies closely follows the work of Dührssen [7]. First, a parameter space $(\mathbf{x})$ is formed of Higgs couplings together with additional partial widths to allow for undetected Higgs decays and additional contributions to the loop-induced Higgs couplings to photon pairs or gluon pairs due to non-SM particles running in the loops. We assume that the measured values correspond to the SM expectations for the purpose of determining statistical uncertainties, then form a $\log$ likelihood function, $L(\mathbf{x})$, which, for a given integrated luminosity, is based on the expected Poisson errors of the channels listed in Sec. 2 and on estimated systematic errors [7], which are tabulated in the Appendix.

As an alternative, in particular for the specific MSSM scenarios discussed in Sec. 4, we use a Gaussian approximation to the log likelihood function, i.e., a $\chi^{2}$ function constructed from the same error assumptions that enter the log likelihood function. We take each of the channels considered in Ref. [7] as a bin in the $\chi^{2}$. To mimic the effect of Poisson statistics on channels with low numbers of events, we discard any channel with $\leq 5$ total events (signal plus background) in both approaches. This is relevant only in the case of low luminosity data. We have checked that the resulting accuracy estimates for coupling measurements are consistent for the two approaches.

Relative to SM expectations, we compute the variation of either $2 L(\mathbf{x})$ or $\chi^{2}(\mathbf{x})$ on this parameter space and trace out the surface of variations by one unit. The $1 \sigma$ uncertainties on each parameter are determined by finding the maximum deviation of that parameter from its $\mathrm{SM}$ value that lies on the $\Delta \chi^{2}=1(\Delta L=1 / 2)$ surface. We repeat the procedure for each Higgs mass value in the range $110 \leq m_{H} \leq 190 \mathrm{GeV}$ in steps of $10 \mathrm{GeV}$.

We perform the fits under three luminosity assumptions for the LHC:

1. $30 \mathrm{fb}^{-1}$ at each of two experiments, denoted $\underline{2 \times 30 \mathrm{fb}^{-1}}$;

2. $300 \mathrm{fb}^{-1}$ at each of two experiments, of which only $100 \mathrm{fb}^{-1}$ is usable for WBF channels at each experiment, denoted $\underline{2 \times 300+2 \times 100 \mathrm{fb}^{-1}}$;

3. $300 \mathrm{fb}^{-1}$ at each of two experiments, with the full luminosity usable for WBF channels, denoted $2 \times 300 \mathrm{fb}^{-1}$.

The second case allows for possible significant degradation of the WBF channels in a high luminosity environment, while the third case shows the benefits of additional improvements in WBF studies at high luminosity. 
In both cases the Higgs boson mass is not fitted, i.e. it is assumed that the mass of the Higgs boson can be measured with high precision $\left(\Delta m_{H} / m_{H}<1 \%\right)$ in $H \rightarrow Z^{(*)} Z^{(*)} \rightarrow$ $4 \ell$ or $H \rightarrow \gamma \gamma$. If both of these channels go unobserved, the theoretical calculations of Higgs boson BRs get a large error due to the relatively low precision and larger systematic errors of mass measurements in WBF $H \rightarrow \tau \tau$ or $H \rightarrow W W$. This is in itself not a problem for the measurement of products of Higgs boson couplings, but a comparison to theoretical prediction, i.e. the upper limit on $\Gamma_{V}$, will suffer considerably from this additional uncertainty.

\subsection{General multi-Higgs-doublet model fits}

We begin by fitting for the uncertainties in the Higgs couplings-squared in the most general scenario that we consider: we assume only that

$$
\begin{aligned}
& g^{2}(H, W)<1.05 \cdot g^{2}(H, W, \mathrm{SM}) \\
& g^{2}(H, Z)<1.05 \cdot g^{2}(H, Z, \mathrm{SM}) .
\end{aligned}
$$

Any model that contains only Higgs doublets and singlets will satisfy the relations without the factor 1.05; the extra 5\% margin allows for theoretical uncertainties in the translation between couplings-squared and partial widths, and also for small admixtures of exotic Higgs states, like $\mathrm{SU}(2)$ triplets. We allow for the possibility of additional particles running in the loops for $H \rightarrow \gamma \gamma$ and $g g \rightarrow H$, fitted by a positive or negative new partial width to these contributions. Additional light hadronic decays of the Higgs boson are fitted with a partial width for undetected decays. (Invisible decays, e.g. to neutralinos could still be observable [38].)

The results for the constraints on the new partial widths are shown in Fig. 1 as a function of Higgs mass for the $2 \times 30 \mathrm{fb}^{-1}$ and $2 \times 300+2 \times 100 \mathrm{fb}^{-1}$ luminosity scenarios and SM rates observed. The new partial width for $H \rightarrow \gamma \gamma$ is most tightly constrained for $120 \lesssim m_{H} \lesssim 140$ $\mathrm{GeV}$, being less than $\pm(25-35) \%$ of $\Gamma_{\gamma}^{\mathrm{SM}}$ for $2 \times 30 \mathrm{fb}^{-1}$ and $\pm(10-15) \%$ for $2 \times 300+$ $2 \times 100 \mathrm{fb}^{-1}$. The new partial width for $g g \rightarrow H$ is less well constrained, being less than $\pm(30-90) \%$ of $\Gamma_{g}^{\mathrm{SM}}$ for $2 \times 30 \mathrm{fb}^{-1}$ and $\pm(30-45) \%$ for $2 \times 300+2 \times 100 \mathrm{fb}^{-1}$ over the whole range of Higgs masses.

The undetected partial width can be constrained to be less than $15-55 \%$ of the total fitted Higgs width for $2 \times 30 \mathrm{fb}^{-1}$ and $15-30 \%$ for $2 \times 300+2 \times 100 \mathrm{fb}^{-1}$, at the $1 \sigma$ level. This undetected partial width is most tightly constrained for Higgs masses above $160 \mathrm{GeV}$.

The resulting precisions on the Higgs boson couplings squared are shown in Fig. 2 as a function of Higgs mass for the same luminosity scenarios, $2 \times 30 \mathrm{fb}^{-1}$ and $2 \times 300+$ $2 \times 100 \mathrm{fb}^{-1}$, and SM rates observed. For the latter case, typical accuracies range between 20 and $40 \%$ for Higgs masses below $150 \mathrm{GeV}$. Above $W$-pair threshold the measurement of the then-dominant $H \rightarrow W W, Z Z$ partial widths improves to the $10 \%$ level. The case of $2 \times 300 \mathrm{fb}^{-1}$ yields only small improvements over the right-hand panel in Fig. 2, except in the case of $g^{2}(H, \tau)$ which shows moderate improvement. However, since this happens for Higgs masses below $\sim 140 \mathrm{GeV}$, this effect can be relatively important in the case of MSSM analyses, see Sec. 4. This can be understood because the $H \rightarrow \tau \tau$ decay is measured only in WBF, and $g(H, \tau)$ does not have a large effect on the Higgs total width or loop-induced couplings. 

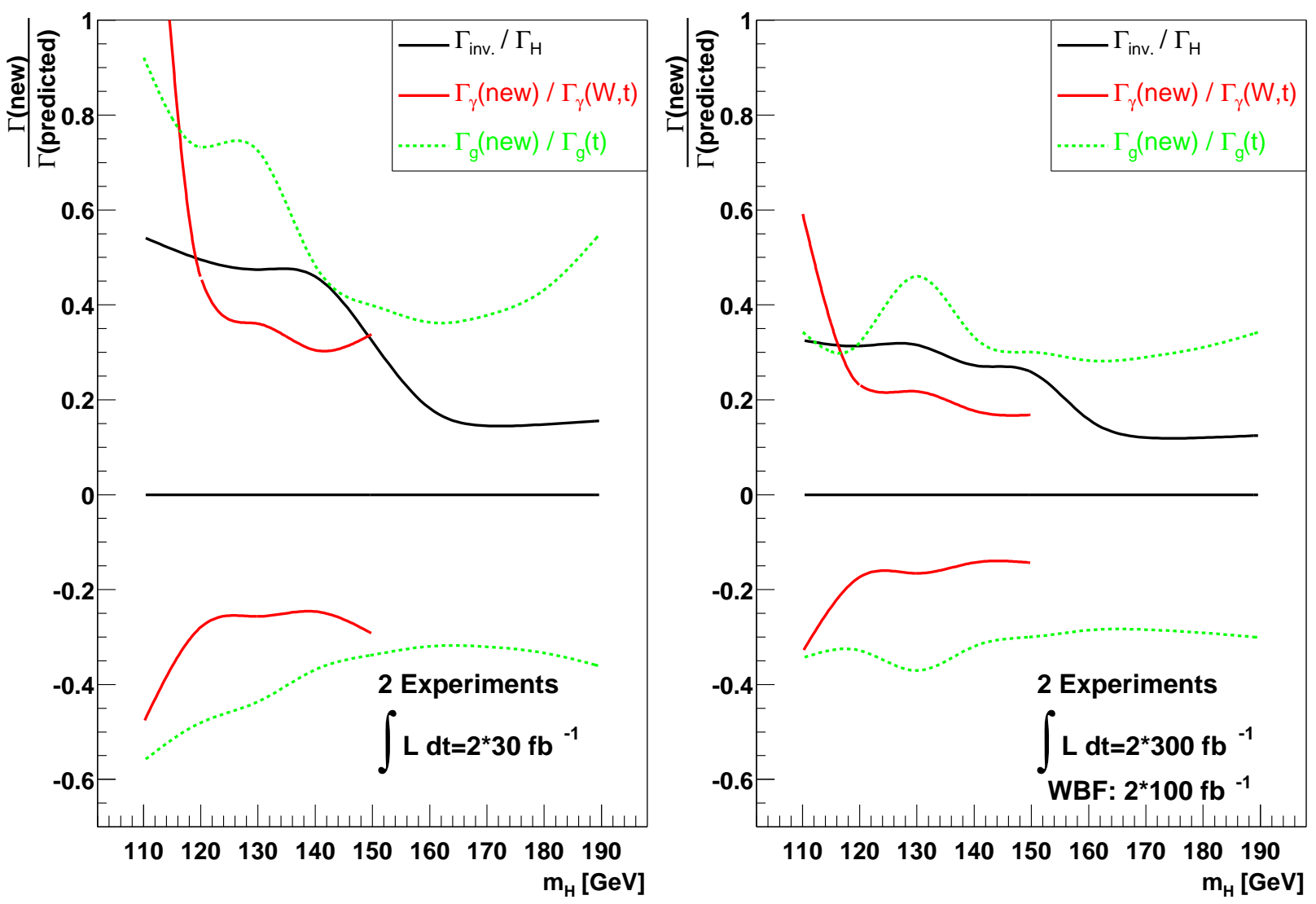

Figure 1: Relative precisions of fitted new partial widths as a function of the Higgs boson mass assuming SM rates and $30 \mathrm{fb}^{-1}$ at each of two experiments (left) and $300 \mathrm{fb}^{-1}$ at each of two experiments for all channels except WBF, for which $100 \mathrm{fb}^{-1}$ is assumed (right). The new partial width can be due to new particles in the loops for $H \rightarrow \gamma \gamma$ and $g g \rightarrow H$ or due to unobservable decay modes. See text for details. Here we make the weak assumption that $g^{2}(H, V)<1.05 \cdot g^{2}(H, V, \mathrm{SM})(V=W, Z)$.

\subsection{Dominant systematic uncertainties}

The results shown in Fig. 2 reflect present understanding of detector effects and systematic errors (see also the Appendix). One should note that improved selection and higher acceptance will decrease the statistical errors. At least as important is work on the reduction of systematic errors. In Fig. 2, the thin lines show expectations with vanishingly small systematics: systematic errors contribute up to half the total error, especially at high luminosity.

For a Higgs boson mass below $140 \mathrm{GeV}$ the main contribution to the systematic uncertainty is the background normalization from sidebands. The largest contribution is from $H \rightarrow b \bar{b}$. For this channel the signal to background ratio is between $1: 4$ and $1: 10$. For the background normalization we assume a systematic error of $10 \%$ [27]. This leads to a huge total systematic error on the measurement of $\Gamma_{b}$, which is the main contribution to the total width $\Gamma$ (the $\mathrm{BR}(H \rightarrow b \bar{b})$ is between $80 \%$ and $30 \%$ ). But a measurement of absolute couplings needs $\Gamma$ as input (see discussion in the introduction to this section), so all measurements of couplings share the large systematic uncertainty on $H \rightarrow b \bar{b}$. 

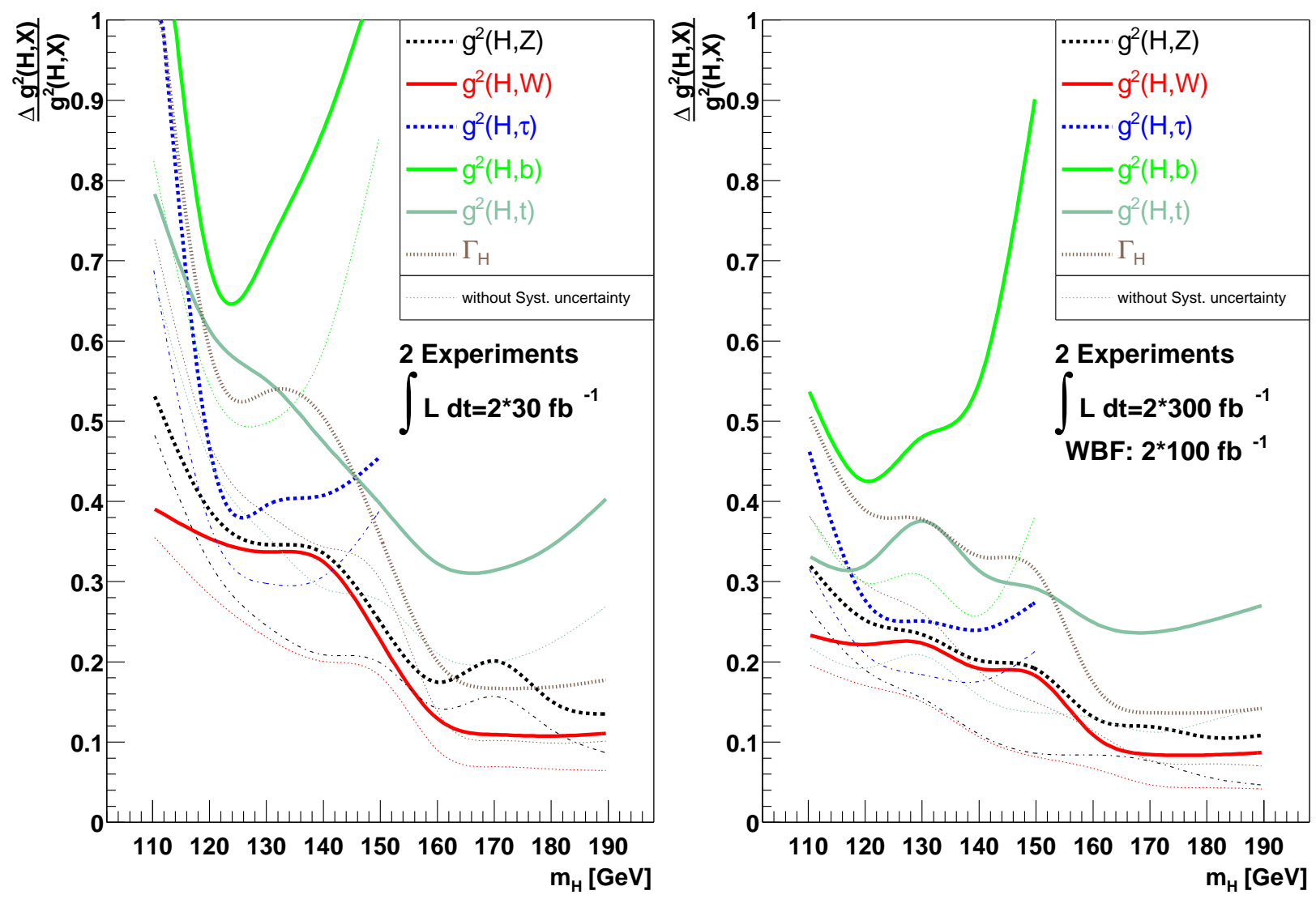

Figure 2: Relative precision of fitted Higgs couplings-squared as a function of the Higgs boson mass for the $2 \times 30 \mathrm{fb}^{-1}$ (left) and the $2 \times 300+2 \times 100 \mathrm{fb}^{-1}$ (right) luminosity scenarios for SM rates. Here we make the weak assumption that $g^{2}(H, V)<1.05 \cdot g^{2}(H, V, \mathrm{SM})(V=W, Z)$ but allow for new particles in the loops for $H \rightarrow \gamma \gamma$ and $g g \rightarrow H$ and for unobservable decay modes. See text for details.

For a Higgs boson mass above $150 \mathrm{GeV}$ there are two dominant contributions to the systematic error:

- the background normalizations in GF, WBF and $t \bar{t} H$ (systematic error $5 \%$ to $15 \%$ )

- the QCD uncertainty in the cross section calculations for GF (20\%) and $t \bar{t} H(15 \%)$ from given Higgs boson couplings.

This is especially evident in the measurement of the top coupling based on the $t \bar{t} H$ channel. Here the systematic uncertainties contribute half of the total error.

\subsection{Additional constraints: SU(2) and SM loops}

The theoretical constraints used so far have been very moderate. If, in addition to the requirement that $g^{2}(H, W)<1.05 \cdot g^{2}(H, W, \mathrm{SM})$ and $g^{2}(H, Z)<1.05 \cdot g^{2}(H, Z$, SM), we assume that no new non-SM particles run in the loops for $H \rightarrow \gamma \gamma$ and $g g \rightarrow H$ (which is approximately fulfilled for the MSSM with a not too-light spectrum), the precision of the coupling measurements improves only slightly, with the only noticeable improvement for Higgs masses below $120 \mathrm{GeV}$. 

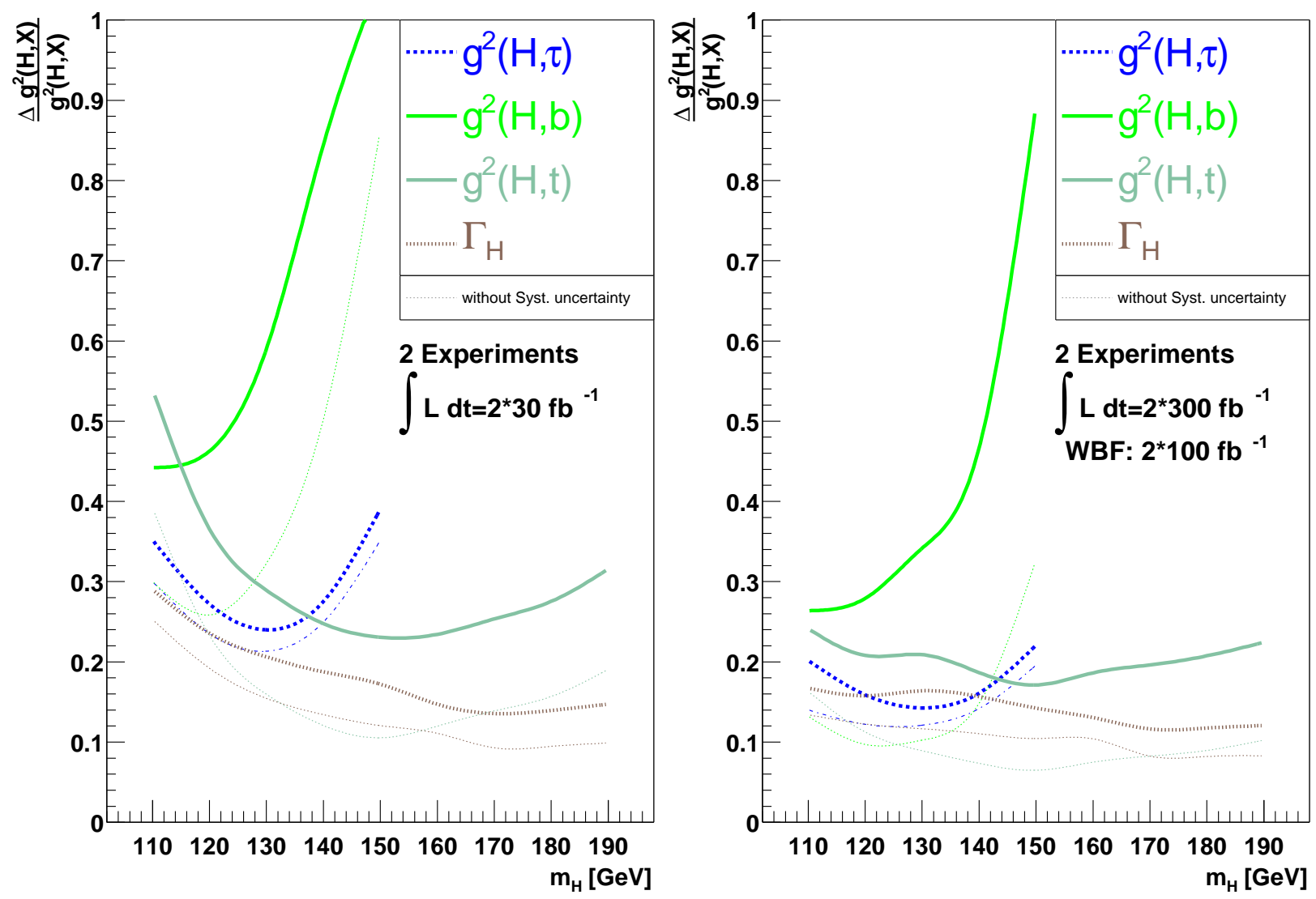

Figure 3: As in Fig. 2, but with more restrictive assumptions. Here we assume that $g^{2}(H, W)=g^{2}(H, W, \mathrm{SM}) \pm 5 \%$ and $g^{2}(H, W) / g^{2}(H, Z)=g^{2}(H, W, \mathrm{SM}) / g^{2}(H, Z, \mathrm{SM}) \pm 1 \%$. We also assume that no new particles run in the loops for $H \rightarrow \gamma \gamma$ and $g g \rightarrow H$, so that these couplings are fixed in terms of the couplings of the SM particles in the loops. As in Fig. 2. additional decays of the Higgs boson are fitted with a partial width for undetected decays (not shown).

Another small improvement comes about by restricting the $W$ and $Z$ couplings to their SM ratio. Within the multi-Higgs-doublet models considered throughout, $\mathrm{SU}(2)$ symmetry relates these two couplings. It thus is natural to forgo an independent measurement of their ratio and to rather assume that

$$
g^{2}(H, W) / g^{2}(H, Z)=g^{2}(H, W, \mathrm{SM}) / g^{2}(H, Z, \mathrm{SM}) \pm 1 \% .
$$

Within the MSSM, this coupling ratio is indeed very close to its SM value.

Over most of the MSSM parameter space even the individual $h V V$ couplings will be close to their SM values since decoupling sets in rapidly once the mass of the $C P$-odd Higgs boson starts to become large, $M_{A} \gtrsim 200 \mathrm{GeV}$. This motivates a fit where in addition to Eq. [1 we assume

$$
g^{2}(H, W)=g^{2}(H, W, \mathrm{SM}) \pm 5 \% .
$$

We again assume that no new non-SM particles run in the loops for $H \rightarrow \gamma \gamma$ and $g g \rightarrow H$. However, we fit additional Higgs boson decays with a partial width for undetected decays. The constraints on this undetected partial width are essentially the same as in our leastconstrained fit, see Fig. [. The resulting parameter precisions are shown in Fig. [3 and 
reach $10-20 \%$ over the entire intermediate Higgs mass range for the $2 \times 300+2 \times 100 \mathrm{fb}^{-1}$ luminosity scenarios. The only exception is $g^{2}(H, b)$, which can be measured only to about $30 \%$ for $m_{H} \lesssim 130 \mathrm{GeV}$.

Relaxing assumptions slightly, by allowing non-SM particles to contribute to the $H \rightarrow \gamma \gamma$ partial width, has a noticeable effect on the coupling determination only for $m_{H} \lesssim 120 \mathrm{GeV}$. For example, for the $2 \times 300+2 \times 100 \mathrm{fb}^{-1}$ luminosity scenario, the precision on $g^{2}(H, \tau)$, $g^{2}(H, b)$ and the Higgs total width at $m_{H}=110 \mathrm{GeV}$ jump to about $40 \%$.

\section{Higgs couplings within the MSSM}

If the obtained Higgs boson couplings differ from the SM predictions, one can investigate at what significance the SM can be excluded from LHC measurements in the Higgs sector alone. As a specific example of physics beyond the SM, we consider the MSSM.

If supersymmetric partners of the SM particles were detected at the LHC, this would of course rule out the SM. It would nevertheless be of interest in such a situation to directly verify the non-SM nature of the Higgs sector. Besides the possible detection of the additional states of an extended Higgs sector, a precise measurement of the couplings of the lightest (SM-like) Higgs boson will be crucial.

For the sake of brevity let us assume that the pseudoscalar Higgs and the charged Higgs are fairly heavy $\left(M_{A} \gtrsim 150 \mathrm{GeV}\right.$, and they may, but need not, have been observed directly) so that they do not interfere with the $h$ signal extraction. We furthermore assume that only decays into SM particles are detected. Then the light Higgs that we consider here will have couplings to the $W$ and $Z$ which are suppressed by the same factor $\sin (\alpha-\beta)$ compared to SM strength, and Higgs couplings to fermions in addition depend on $\tan \beta=v_{2} / v_{1}$ and $\Delta_{b}$ [39], which incorporates non-universal loop corrections to the $h \bar{b} b$ coupling. A fit of the Higgs couplings can then be performed in terms of this reduced parameter set. Obviously this analysis falls within the $g_{V} \leq g_{V}^{\mathrm{SM}}$ analysis described in the previous section. Upper bounds on the expected measurement errors for MSSM partial widths can hence be derived from Fig. 2, while Fig. 3 gives an estimate of errors which can be expected for $M_{A} \gtrsim 200 \mathrm{GeV}$, for which the Higgs couplings to $W$ and $Z$ bosons have sufficiently approached their SM values.

A quantitative, global measure of how well the LHC can distinguish the SM from a specific MSSM scenario is provided by a $\chi^{2}$-analysis of the deviations expected in that case. As a first example we consider the $m_{h}^{\max }$ scenario of Ref. [8]. We calculate the mass and branching fractions of the MSSM Higgs boson using HDECAY3.0 [40], using the FeynHiggsFast1.2.2 $[41,42]$ option to compute the MSSM Higgs masses and couplings. Assuming that, for a given $M_{A}$ and $\tan \beta$, the corresponding SUSY model is realized in nature, we may ask at what significance the SM would be ruled out from $h$ measurements alone. We examine MSSM points only for $M_{A}>150 \mathrm{GeV}$, where the narrow width approxmation is still valid. The resulting contours are shown in Fig. 4 for the three luminosity assumptions defined in Sec. 3.1. In the areas to the left of the contours the SM can be rejected with more than $5 \sigma$ or $3 \sigma$ significance, respectively.

The $\chi^{2}$ definition in Fig. 4 assumes the same systematic errors as our analysis in Sec. 3 , Event rates and resulting statistical errors, however, are those expected for the MSSM.

The source of the MSSM analysis sensitivity can be understood as follows. In the $m_{h}^{\max }$ scenario for $M_{A} \gtrsim 200 \mathrm{GeV}$, the couplings of $h$ to SM particles all essentially obtain their $\mathrm{SM}$ values except for the $h b b$ and $h \tau \tau$ couplings, due to the slower decoupling behavior of 

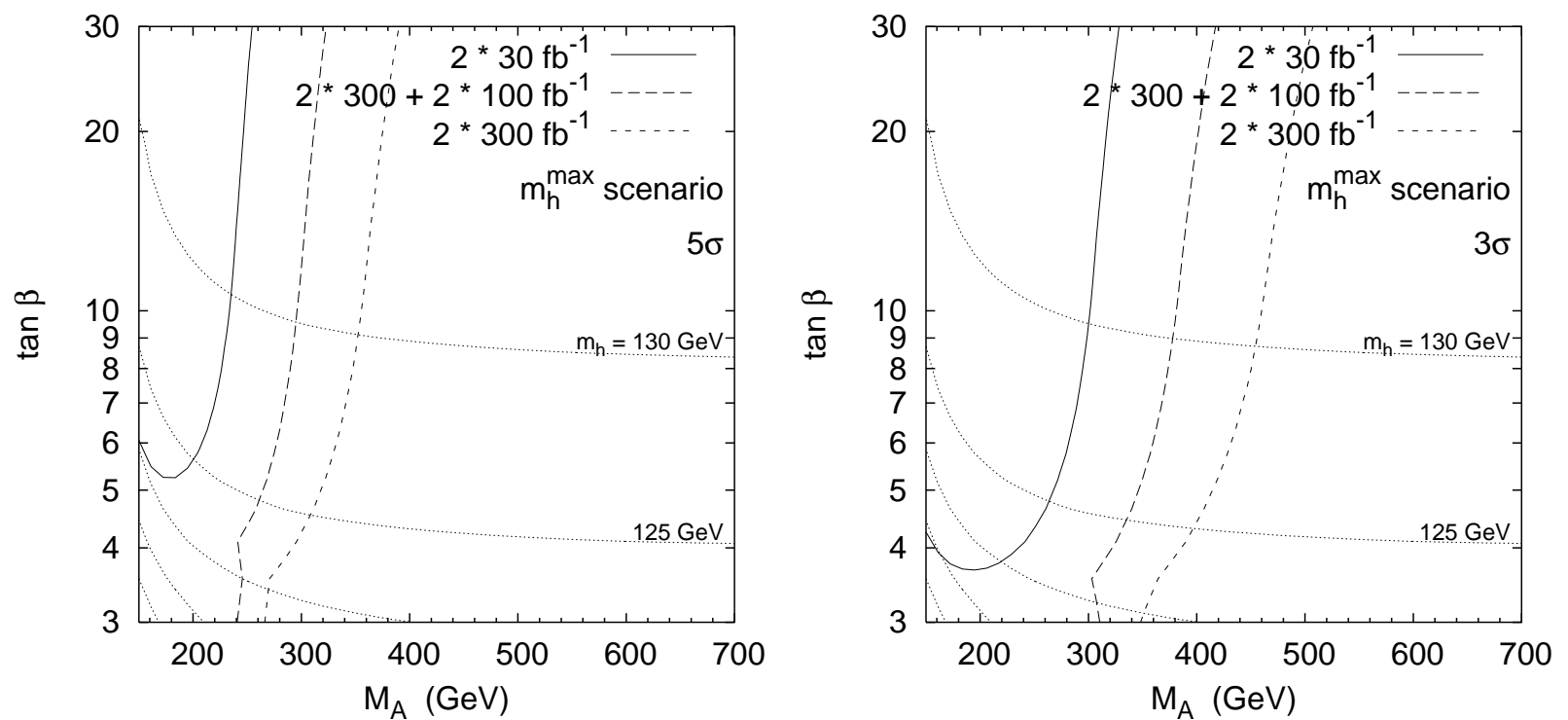

Figure 4: Fit within the MSSM $m_{h}^{\max }$ scenario in the $M_{A}-\tan \beta$ plane for three luminosity scenarios. The two panels show the region (to the left of the curves) which would yield a $\geq 5 \sigma\left(\Delta \chi^{2} \geq 25\right)$ or $\geq 3 \sigma\left(\Delta \chi^{2} \geq 9\right)$ discrepancy from the SM. The mostly-horizontal dotted lines are contours of $m_{h}$ in steps of $5 \mathrm{GeV}$.

the latter. In this scenario the SUSY threshold corrections to the $b$ mass are also quite small, so that the ratio of the $h b b$ and $h \tau \tau$ couplings essentially takes its SM value. The $h \rightarrow b \bar{b}$ decay mode dominates the Higgs total width in this scenario. The pattern of Higgs coupling deviations can then be summarized as follows: all the Higgs production cross sections considered in our study are SM-like; the partial widths into $b \bar{b}$ and $\tau \tau$ are equally enhanced (but with SM-like BRs since the total width is dominated by $b \bar{b}$ and $\tau \tau$ decays). This results in a larger total width for the Higgs boson. The branching ratios into all other final states $\left(W W^{*}, Z Z^{*}, \gamma \gamma\right)$ are smaller than in the SM, reflecting this total width enhancement.

We focus on the WBF channels, which have the strongest impact on the MSSM fit. If systematic errors could be neglected, the well-measured WBF $q q H \rightarrow q q W W^{*}$ channel would give the best sensitivity to the discrepancy from the SM, since it is sensitive to the Higgs branching ratio into $W W^{*}$. The less-well-measured WBF $q q H \rightarrow q q Z Z^{*}, q q \gamma \gamma$ channels could be added to increase the statistics. However, the systematic uncertainties on the luminosity (5\%), WBF cross section (4\%), and forward tagging/veto jets (5\%) hide this sensitivity to the Higgs coupling deviations. These systematic uncertainties can be brought under control by including the WBF $q q H \rightarrow q q \tau \tau$ channel in the fit. While this channel by itself provides no sensitivity to the deviation from the SM (because the WBF cross section and the branching ratio to taus are both SM-like), it serves to normalize out the systematic uncertainties. To a first approximation, this can be thought of as taking the ratio of the WBF rates with Higgs decays to $W W^{*}\left(Z Z^{*}, \gamma \gamma\right)$ versus $\tau \tau$, in which the aforementioned systematic uncertainties cancel. The $\chi^{2}$ fit of the rates in these channels offers a slight improvement over the ratio method because the systematic uncertainties are somewhat better under control.

In Fig. 5 we analyze the impact of the different channels included in our analysis. Within 


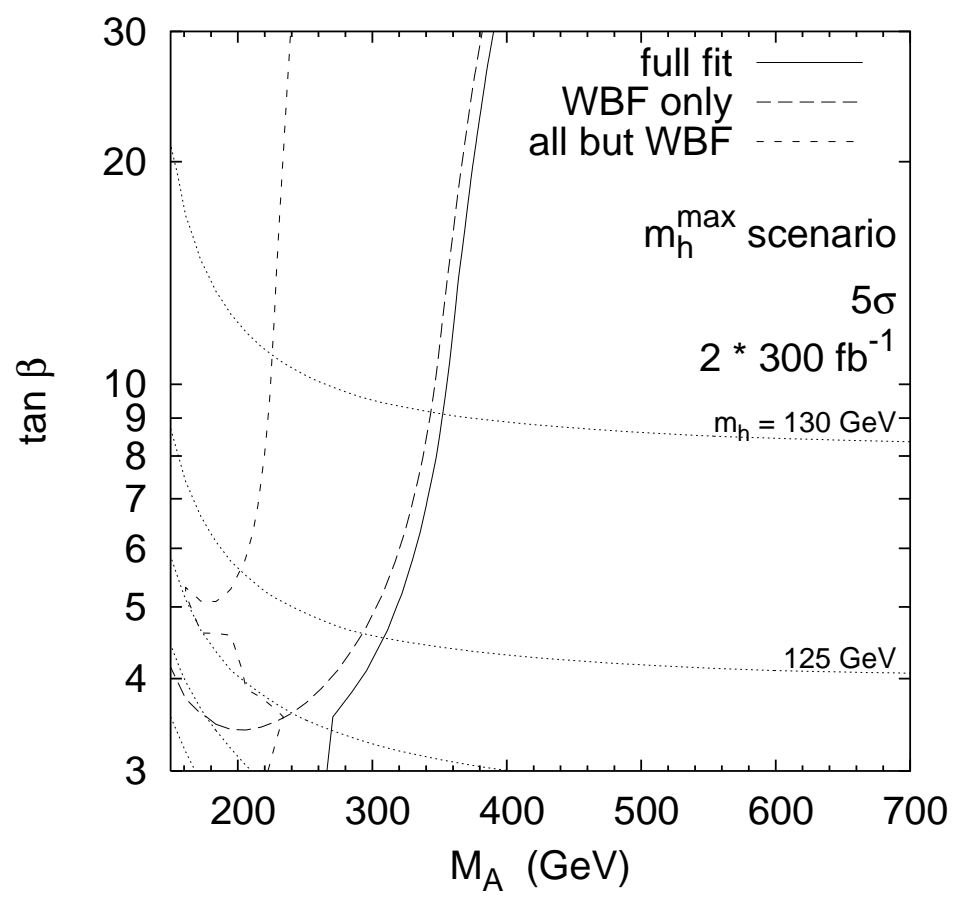

Figure 5: Fit within the MSSM $m_{h}^{\max }$ scenario in the $M_{A}-\tan \beta$ plane for the $2 \times 300 \mathrm{fb}^{-1}$ luminosity scenario. The $5 \sigma$ lines are shown using WBF channels only (long-dashed), all channels except WBF (short-dashed), and the full fit (solid line).

the $m_{h}^{\max }$ scenario we show the $5 \sigma$ contours in the $2 \times 300 \mathrm{fb}^{-1}$ luminosity scenario in the $M_{A}-\tan \beta$ plane. We plot separately the fit using only the WBF channels (long-dashed), using all channels except WBF (short-dashed), and the full fit (solid). It becomes obvious that the WBF channels have the strongest impact. Therefore it will be very helpful for this kind of analysis if the WBF channels can be fully exploited at high luminosity running.

Finally we would like to emphasize that the contours appear significantly different for different SUSY scenarios: other SUSY parameters can have a large effect on the relation of $m_{h}, M_{A}, \tan \beta$ and Higgs couplings. This is shown in Fig. 6, where the $3 \sigma$ significance curves are shown in the "gluophobic Higgs" (left) and the "small $\alpha_{\text {eff" }}$ (right) scenarios [8]. In the gluophobic Higgs scenario the ggh coupling is strongly suppressed over the whole $M_{A}-\tan \beta$ plane due to additional contributions from scalar top loops. We note that for this scenario the $5 \sigma$ curves (not shown) move significantly to the left. Within the small $\alpha_{\text {eff }}$ scenario loop corrections can suppress the $h b b$ and $h \tau \tau$ couplings for moderate values of $M_{A}$ and large values of $\tan \beta$. Both effects yield a strong deviation from the corresponding $3 \sigma$ curves obtained in the $m_{h}^{\max }$ scenario.

It should be noted that the shown sensitivity to $M_{A}$ cannot directly be translated into indirect bounds on $M_{A}$. To establish realistic bounds, a careful analysis of the experimental errors arising from the incomplete knowledge of the spectrum of supersymmetric particles and of the theoretical uncertainties from unknown higher-order corrections is necessary. However, we observe that the contours shift only slightly if one instead uses SM rates to calculate the statistical errors. 

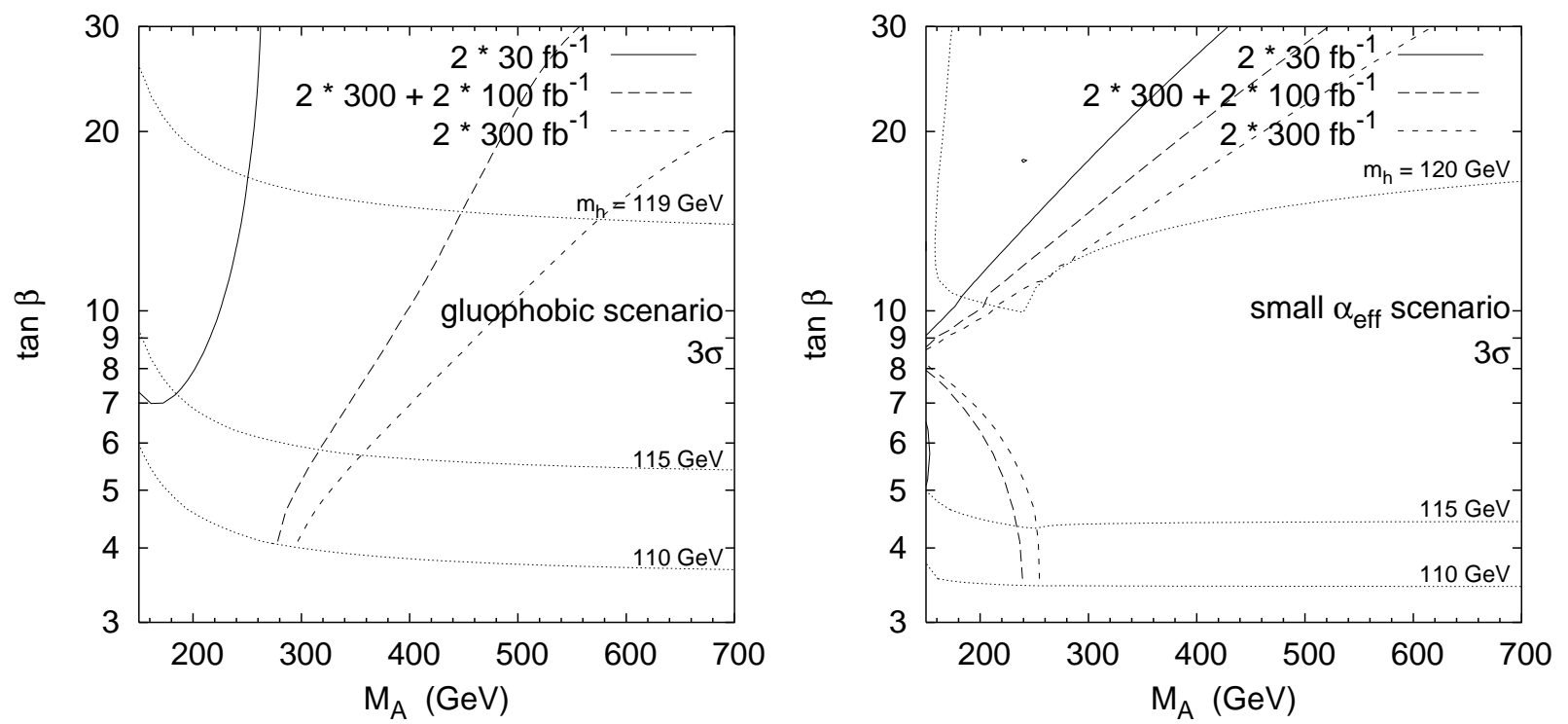

Figure 6: Fit within the MSSM "gluophobic Higgs" (left) and the "small $\alpha_{\text {eff }}$ (right) scenario (see text) in the $M_{A}-\tan \beta$ plane for three luminosity scenarios. The two panels show the region (to the left of the curves) in which a $\geq 3 \sigma\left(\Delta \chi^{2} \geq 9\right)$ discrepancy from the SM would be observed. The mostly-horizontal dotted lines are contours of $m_{h}$, where the masses are indicated.

\section{$5 \quad$ Summary and outlook}

Measurements of the Higgs sector are expected to provide many complementary signatures after several years of LHC running. Combining these measurements allows one to extract information on Higgs partial widths and Higgs couplings to fermions and gauge bosons. Because significant contributions from unobservable channels cannot easily be ruled out at the LHC, model-independent analyses produce large correlations between extracted partial widths. A reduction of correlations and hence smaller errors on particular couplings can be achieved with only very weak theory assumptions applicable to quite general Higgs sector scenarios. In this paper we have analyzed the constraints expected in generic multi-Higgsdoublet models, namely that $H V V$ couplings cannot be larger than within the SM. Within such models, the LHC can measure Higgs couplings to the top quark, tau lepton, and $W$ and $Z$ bosons with accuracies in the $10-40 \%$ range once $300 \mathrm{fb}^{-1}$ of data have been collected. If, on the other hand, the SLHC will be realized, one could hope for significant improvements over the results presented here. This applies in particular for the bottom Yukawa coupling determination.

Within the MSSM, significant deviations in the Higgs sector should be observable at the LHC, provided that the charged and the pseudoscalar Higgs masses are not too heavy, i.e., that decoupling is not completely realized. For example, within the $m_{h}^{\max }$ scenario and with $300 \mathrm{fb}^{-1}$ of data, the LHC can distinguish the MSSM and the SM at the $3 \sigma$ level up to $M_{A} \simeq 450 \mathrm{GeV}$ and with $5 \sigma$ significance up to $M_{A} \simeq 350 \mathrm{GeV}$ with the Higgs data alone. The LHC will thus provide a surprisingly sensitive first look at the Higgs sector, even though it cannot match the precision and model-independence of analyses which are expected for a linear $e^{+} e^{-}$collider [43-45]. 
So far we have investigated the situation where no important channel suffers substantial suppression. However, it might be (within Supersymmetry or another extension of the SM) that the WBF channels are degraded, or that the Higgs decays more strongly to unobservable $c \bar{c}$ or $g g$ final states. Other decays like $h \rightarrow \tilde{\chi}_{1}^{0} \tilde{\chi}_{1}^{0}[38]$ or $h \rightarrow \mu^{+} \mu^{-}$[46] may be detectable, or upper bounds may be put on their partial widths. Channels with low statistics might be absent. Finally, the mass measurement might be less precise due to a suppression of $H \rightarrow \gamma \gamma$, thus weakening the Higgs mass constraint. These scenarios are beyond the scope of this paper and will be discussed in a forthcoming publication.

\section{Acknowledgments}

We would like to thank M. Carena for useful discussions during early stages of this work. H.L. and D.Z. were supported in part by the U.S. Department of Energy under grant DEFG02-95ER40896 and in part by the Wisconsin Alumni Research Foundation. This work was also supported by the European Community's Human Potential Programme under contract HPRN-CT-2000-00149 Physics at Colliders. S.H. thanks the DESY theory division for kind hospitality in the final stages of this work.

\section{Appendix: systematic uncertainies}

The systematic errors include uncertainties on luminosity and detector effects which are summarized in Tab. 1. All these numbers are estimates. More definite numbers will be known only once the LHC experiments are running.

\begin{tabular}{|lll|}
\hline$L$ & $5 \%$ & Measurement of luminosity \\
\hline$\epsilon_{D}$ & $2 \%$ & Detector efficiency \\
\hline$\epsilon_{L}$ & $2 \%$ & Lepton reconstruction efficiency \\
\hline$\epsilon_{\gamma}$ & $2 \%$ & Photon reconstruction efficiency \\
\hline$\epsilon_{b}$ & $3 \%$ & b-tagging efficiency \\
\hline$\epsilon_{\tau}$ & $3 \%$ & hadronic $\tau$-tagging efficiency \\
\hline$\epsilon_{\text {Tag }}$ & $5 \%$ & WBF tag-jets / jet-veto efficiency \\
\hline$\epsilon_{\text {Iso }}$ & $3 \%$ & Lepton isolation $(H \rightarrow Z Z \rightarrow 4 \ell)$ \\
\hline
\end{tabular}

Table 1: Estimated systematic uncertainties on luminosity and detector effects, see e.g. Ref. [7].

The systematic background normalization uncertainties of the individual channels are split into two components, shown in the second and third column of Tab. 2. The first part is the uncertainty on the shape of the background derived from extrapolating a perfectly measured sideband into the signal region. The second part is needed to estimate the statistical error on the measurement of the sideband itself. We used this manner of estimating the number of events in the sideband since actual numbers for sidebands are not contained in the existing analyses.

The uncertainties in Tab. 3 summarize the theoretical QCD and PDF uncertainties on Higgs boson production. For the WBF channels there is an additional 10\% (after applying 


\begin{tabular}{|l|r|r|}
\hline \multicolumn{1}{|c|}{ Decay } & Shape & $N_{N} / N_{B}$ \\
\hline$H \rightarrow Z Z^{(*)} \rightarrow 4 l$ & $1 \%$ & 5 \\
\hline$H \rightarrow W W^{(*)} \rightarrow \ell \nu \ell \nu$ & $5 \%$ & 1 \\
\hline$H \rightarrow \gamma \gamma$ & $0.1 \%$ & 10 \\
\hline$H \rightarrow \tau \tau$ & $5 \%$ & 2 \\
\hline$H \rightarrow b b$ & $10 \%$ & 1 \\
\hline
\end{tabular}

Table 2: Estimated systematic uncertainties on background normalization. The extrapolation of a measured sideband into the signal region results in an uncertainty on the extrapolated number of background events. This contribution is given in the second column as systematic error on the shape. The third column shows the ratio $N_{N} / N_{B}$ where $N_{N}$ is the actual number of events in the sideband and $N_{B}$ is the expected number of background events in the signal region. The ratio is used to estimate the statistical error on the measurement of the sideband itself from the numbers $N_{B}$ given in the experimental studies.

the minijet veto) contribution from $g g \rightarrow H g g$ [37], which has its own theory uncertainty of a factor of 2 .

\begin{tabular}{|l|l|}
\hline $\mathrm{GF}$ & $20 \%$ \\
\hline$t \bar{t} H$ & $15 \%$ \\
\hline$W H$ & $7 \%$ \\
\hline$Z H$ & $7 \%$ \\
\hline $\mathrm{WBF}$ & $4 \%$ \\
\hline$g g \rightarrow H g g$ & $100 \%$ \\
\hline
\end{tabular}

Table 3: Theoretical QCD and PDF uncertainties on the various Higgs boson production channels. The channel $g g \rightarrow H g g$ was added to all WBF analyses at $10 \%$ of the WBF rate with an uncertainty of a factor 2 .

\section{References}

[1] R. Barate et al. [LEP Higgs working group], Phys. Lett. B 565 (2003) 61 arXiv:hep-ex/0306033.

[2] M. W. Grünewald, arXiv:hep-ex/0304023; updated as: S. Roth, talk given at "Rencontres de Moriond: QCD and Hadronic interactions", La Thuile (Italy), March 28 - April 4 2004, see: moriond.in2p3.fr/QCD/2004/ThursdayAfternoon/Roth.pdf;

see also: lepewwg. web. cern. ch/LEPEWWG/Welcome.html.

[3] P. Garcia-Abia and W. Lohmann, Eur. Phys. J. directC 2 (2000) 2 arXiv:hep-ex/9908065.

[4] A. Djouadi et al., arXiv:hep-ph/0002258.

[5] D. Zeppenfeld, R. Kinnunen, A. Nikitenko and E. Richter-Was, Phys. Rev. D 62 (2000) 013009 arXiv:hep-ph/0002036.

[6] A. Belyaev and L. Reina, JHEP 0208, 041 (2002) arXiv:hep-ph/0205270. 
[7] M. Dührssen, ATL-PHYS-2003-030, available from http://cdsweb.cern.ch.

[8] M. Carena, S. Heinemeyer, C. E. M. Wagner and G. Weiglein, Eur. Phys. J. C 26 (2003) 601 arXiv:hep-ph/0202167.

[9] R. V. Harlander and W. B. Kilgore, Phys. Rev. Lett. 88 (2002) 201801 arXiv:hep-ph/0201206.

[10] C. Anastasiou and K. Melnikov, Nucl. Phys. B 646 (2002) 220 arXiv:hep-ph/0207004.

[11] V. Ravindran, J. Smith and W. L. van Neerven, Nucl. Phys. B 665 (2003) 325 arXiv:hep-ph/0302135.

[12] K. Cranmer, B. Mellado, W. Quayle and S. L. Wu, arXiv:hep-ph/0307242.

[13] I. Puljak, Dissertation, Ecole Polytechnique, Palaiseau, France, 2000.

[14] ATLAS detector and physics performance. Technical design report. Vol. 2, CERNLHCC-99-15.

[15] CMS: The electromagnetic calorimeter. Technical design report, CERN-LHCC-97-33.

[16] S. Abdullin et al., Phys. Lett. B 431 (1998) 410 arXiv:hep-ph/9805341.

[17] K. Cranmer, B. Mellado, W. Quayle and S. L. Wu, ATL-COM-PHYS-2003-036, arXiv:hep-ph/0401088.

[18] M. Dubinin, CMS-NOTE-2001/022.

[19] M. Dubinin, V. A. Ilyin and V. I. Savrin, Prepared for 12th International Workshop on High-Energy Physics and Quantum Field Theory (QFTHEP 97), Samara, Russia, 4-10 Sep 1997.

[20] M. Dittmar and H. K. Dreiner, Phys. Rev. D 55 (1997) 167 arXiv:hep-ph/9608317.

[21] D. Green et al., J. Phys. G 26 (2000) 1751.

[22] S. Asai et al., Prospects for the search of a Standard Model Higgs boson in ATLAS using vector boson fusion, EUR. Phys. J. C direct, epjcd/S2003-01-010-8, arXiv:hep-ph/0402254

[23] N. Akchurin et al., CMS-NOTE-2001/016.

[24] J. Leveque, J. B. Vivie, V. Kostioukhine and A. Rozanov, ATL-PHYS-2002-019.

[25] V. Cavasinni and D. Costanzo, ATL-PHYS-2000-013.

[26] G. Azuelos et al., "Search for the standard model Higgs boson using vector boson fusion at the LHC", Prepared for Workshop on Physics at TeV Colliders, Les Houches, France, 21 May - 1 Jun 2001.

[27] J. Cammin and M. Schumacher, ATL-PHYS-2003-024.

[28] K. Jakobs, ATL-PHYS-2000-008. 
[29] G. Eynard, Etude de la production associée du boson de Higgs HW, Htż, HZ $\rightarrow \gamma \gamma+$ $e^{ \pm} / \mu^{ \pm}+X$ avec le détecteur ATLAS, auprès du LHC, PhD thesis, Grenoble (1998).

[30] K. Cranmer, B. Mellado, W. Quayle, S. L. Wu, ATL-COM-PHYS-2003-006.

[31] R. Mazini, G. Azuelos, ATL-COM-PHYS-2002-045.

[32] V. Drollinger, T. Müller and D. Denegri, arXiv:hep-ph/0111312.

[33] D. Green et al., FERMILAB-FN-0705.

[34] V. Drollinger, T. Müller and D. Denegri, arXiv:hep-ph/0201249.

[35] J. Campbell, R. K. Ellis and D. Rainwater, Phys. Rev. D 68 (2003) 094021 arXiv:hep-ph/0308195.

[36] M. Schumacher, talk given at the "Advanced Studies Institute, Physics at LHC", Prague, July 2003, see: asipraha.web.cern.ch/asipraha/2003/LHC/.

[37] V. Del Duca et al., Phys. Rev. Lett. 87, 122001 (2001) arXiv:hep-ph/0105129.

[38] O. J. P. Eboli and D. Zeppenfeld, Phys. Lett. B 495 (2000) 147 arXiv:hep-ph/0009158; R. M. Godbole, M. Guchait, K. Mazumdar, S. Moretti and D. P. Roy, Phys. Lett. B 571, 184 (2003) arXiv:hep-ph/0304137.

[39] L. J. Hall, R. Rattazzi and U. Sarid, Phys. Rev. D 50 (1994) 7048 arXiv:hep-ph/9306309.

[40] A. Djouadi, J. Kalinowski and M. Spira, Comput. Phys. Commun. 108 (1998) 56 arXiv:hep-ph/9704448.

[41] S. Heinemeyer, W. Hollik and G. Weiglein, arXiv:hep-ph/0002213.

[42] S. Heinemeyer, W. Hollik and G. Weiglein, Phys. Lett. B 455 (1999) 179 arXiv:hep-ph/9903404.

[43] J. A. Aguilar-Saavedra et al. [ECFA/DESY LC Physics Working Group Collaboration], arXiv:hep-ph/0106315.

[44] T. Abe et al. [American Linear Collider Working Group Collaboration], in Proc. of the $A P S / D P F / D P B$ Summer Study on the Future of Particle Physics (Snowmass 2001) ed. N. Graf, arXiv:hep-ex/0106056;

[45] K. Abe et al. [ACFA Linear Collider Working Group Collaboration], arXiv:hep-ph/0109166.

[46] T. Plehn and D. Rainwater, Phys. Lett. B 520, 108 (2001) arXiv:hep-ph/0107180;

T. Han and B. McElrath, Phys. Lett. B 528, 81 (2002) arXiv:hep-ph/0201023|;

E. Boos, A. Djouadi and A. Nikitenko, Phys. Lett. B 578, 384 (2004) arXiv:hep-ph/0307079. 